\title{
EUCALYPTUS NITENS COMO MATERIA PRIMA PARA TABLEROS DE PARTICULAS
}

\section{EUCALYPTUS NITENS AS RAW MATERIAL FOR PARTICLEBOARDS}

\author{
Hernán Poblete W, Rodrigo Burgos $\boldsymbol{O}^{I}$.
}

\begin{abstract}
RESUMEN
El objetivo del trabajo fue evaluar la madera de Eucalyptus nitens (Deane et Maiden) Maiden como materia prima para tableros de partículas. Se controlaron la densidad de la madera, las dimensiones de las partículas obtenidas, el coeficiente de esbeltez y el valor $\mathrm{pH}$ de las partículas. Se fabricaron tableros con densidades entre 450 y $750 \mathrm{~kg} / \mathrm{m}^{3}$ y se determinaron las propiedades flexión, tracción perpendicular, densidad de los tableros, hinchamiento y absorción de agua a las 2 y 24 horas. Se calculó el grado de correlación de las distintas propiedades del tablero con la densidad de los mismos. Las características de la madera, de las partículas y las propiedades de los tableros demuestran que la madera de Eucalyptus nitens es apta para ser utilizada en la fabricación de estos aglomerados. A medida que aumenta la densidad del tablero se provoca un aumento en las propiedades mecánicas. El hinchamiento y la absorción de agua mejoran con aumentos de la densidad. El análisis de correlación entre las propiedades dependientes (hinchamiento, absorción, flexión y tracción) y la densidad del tablero arrojó coeficientes de correlación mayores a 0,7. La propiedad crítica es el hinchamiento, por lo que se recomienda agregar una emulsión de cera y un catalizador en el adhesivo de la capa central.
\end{abstract}

Palabras claves: Tablero de partículas, Eucalyptus nitens, densidad de tablero, propiedades físicas y mecánicas.

\section{ABSTRACT}

The aim of the study was to evaluate Eucalyptus nitens as raw material for the production of particleboards. Wood density and particle size, slenderness and $\mathrm{pH}$-value were controlled. Bending strength, internal bond, density, thickness swelling and water absorption after 2 and 24 hours of particleboards with densities between 450 and $750 \mathrm{~kg} / \mathrm{m}^{3}$, were determined. Correlations between board density and properties were calculated. The wood and particle characteristics as well as the obtained board properties proved that Eucalyptus nitens could be used for the particleboard production. As board density increases, higher mechanical properties were founded. Thickness swelling and water absorption decreased as density of particleboard increased. High correlation factors $(>0,7)$ between dependent variables (thickness swelling, absorption, bending strength and IB) and board density were obtained. Due to swelling is a critical property, we recommend the addition of a wax emulsion and a catalyst in the middle layer of the particleboard.

Keywords: Particleboard, Eucalyptus nitens, board density, physical and mechanical properties.

${ }^{1}$ Universidad Austral de Chile. Fac. Cs. Forestales. Valdivia. Chile

Autor para correspondencia: hernanpoblete@uach.cl

Recibido: 21.01.2009 Aceptado: 25.03.2010 


\section{INTRODUCCIÓN}

Debido al rápido crecimiento de Eucalyptus nitens, $>30 \mathrm{~m}^{3} / \mathrm{ha} / \mathrm{año}$, se pueden efectuar cosechas tempranas, entre 8 y 12 años (Poblete 2004). La densidad básica de la madera de E. nitens, aproximada a $510 \mathrm{~kg} / \mathrm{m}^{3}$, indica que esta especie tendría una posibilidad de uso en la fabricación de tableros de partículas (Poblete 2002).

Los resultados de dos experiencias industriales en Chile con E. nitens son auspiciosos (Gorrini et al.2004). En el 2005 ya se informaba de la utilización de esta especie en la producción de tableros OSB certificados para exportar a Norteamérica (Vásquez 2005). El trabajo de Gorrini et al (2004) tiene el valor de ser una aplicación a escala industrial, sin embargo considera una participación de E. nitens en una mezcla, con sólo $20 \%$, lo que no permite asegurar que los resultados con esta especie sean comparables a los que se obtienen con las materias primas tradicionales, aserrín y astillas de pino radiata.

Cuando se planifica fabricar tableros con una nueva especie, hay que considerar las propiedades de la madera que influyen sobre el proceso y estudiar algunas variables de éste que afectan las propiedades del tablero. Entre las propiedades de la madera que tienen un efecto importante en las características del tablero se destacan la densidad y la acidez. Desde el punto de vista del proceso o del producto, la densidad del tablero es una de las más importantes. Cambiando la densidad del tablero se pueden variar las propiedades físicas y mecánicas, lo que permite calcular la densidad necesaria para obtener un producto que cumpla con las exigencias de las normas (Poblete 2001).

Considerando la importancia del efecto de la densidad de los tableros en las propiedades físicas y mecánicas de los mismos, en el presente trabajo se optó por determinar la relación de densidad con otras propiedades en tableros fabricados en un 100\% con Eucalyptus nitens.

Efecto de la densidad de la madera. La densidad de la madera es uno de los factores más importantes en la fabricación de tableros de partículas. Aún cuando no existe una variable que en forma aislada permita definir a una especie como apropiada para fabricar estos tableros, la densidad de la madera es considerada por muchos autores como la que más afecta a las propiedades del tablero (Vital et al 1974, Kollmann et al. 1975).

Al variar la densidad de la madera, la calidad del corte para obtener partículas cambia, lo que trae consigo variaciones en las dimensiones y geometría de las partículas y esto afecta a diversas propiedades del tablero. Al cambiar la geometría de la partícula se varía la forma como se comprime y transmite las propiedades de la madera al tablero, se cambia la superficie específica de la masa de partículas y con ello la distribución del adhesivo. Se puede afectar el aspecto de la superficie del tablero y sus posibilidades de recubrimiento (Poblete 2001).

Si se desea mantener constante las propiedades físicas y mecánicas del tablero y se cambia la densidad de la madera, debe ajustarse la compactación de la estera, razón de compresión (Poblete 2001). Aumentos en la densidad de la madera producen una disminución de prácticamente todas las propiedades mecánicas del tablero (Stegmann y Durst 1964, Moslemi 1974). Las propiedades físicas también se ven afectadas por la variación de la densidad de la madera. Así, la estabilidad dimensional está determinada por la interacción entre la densidad de la especie y la densidad del tablero (Stewart y Lehmann 1973, Poblete et al. 2000).

Efecto de la acidez de la madera, valor de pH. Al variar la especie utilizada, se alteran las propiedades químicas de la mezcla de adhesivo y partículas que se comprime en la prensa (Poblete 2001). La acidez de la madera, toma mayor importancia cuando se trata de adhesivos que fraguan en ambiente ácido, como la urea formaldehído. En este caso la tasa de fraguado del adhesivo es determinada por 
la acidez de la madera. El valor de $\mathrm{pH}$ y la capacidad de equilibrio de ionización está determinada por la cantidad y tipo de extraíbles que posea la madera. El valor de $\mathrm{pH}$ afecta al proceso de fabricación principalmente en el encolado, por lo que necesariamente debe ser controlado. Al tener la madera un pH no compatible con el adhesivo, se afectan las propiedades mecánicas (Roffael et al. 2000).

Efecto de la densidad del tablero. Cuando varía la densidad del tablero se producen alteraciones de todas las propiedades físicas y mecánicas. Si se pretende fabricar un tablero con una nueva especie, esta propiedad es normalmente la primera prioridad en los ajustes del programa de producción. La densidad del tablero está determinada por el grado de compactación de las partículas de madera y es dependiente de la densidad de la madera y del proceso de prensado (Poblete et al. 2000). Este parámetro tiene variaciones dentro de un panel. Se debe tener presente que una reducción en la densidad de los tableros de partículas, trae consigo un aumento de la capacidad de absorción de agua y un descenso en las propiedades mecánicas (Kelly 1977).

\section{MATERIAL Y METODOS}

\section{Materiales}

Madera. Se utilizó madera de Eucalyptus nitens, cosechada en predios de la empresa Forestal Mininco S.A. que se ubican en la zona de Mulchén, VIII Región de Chile. Los 10 árboles utilizados tenían 13 años. La muestra total fue de 20 trozas, las que se descortezaron manualmente en el bosque.

Adhesivo. El adhesivo empleado fue urea-formaldehído, diluida a una concentración del 50\%.

\section{Metodología}

Propiedades de la madera: Con el objetivo de evaluar las propiedades más importantes de la madera como materia prima para tableros de partículas, se determinaron la densidad y acidez de acuerdo con la siguiente metodología:

- Densidad de la madera (DIN 52182) en probetas de la periferia y centro de rodelas tomadas en la base de 10 árboles (40 probetas en total).

- Valor de pH, según Roffael et al (2000). Para determinar el valor de pH se realizaron mediciones en partículas de capa externa e interna, tanto en una mezcla con agua destilada como también en el filtrado obtenido luego de una agitación mecánica por cuatro horas a $250 \mathrm{rpm}$. La relación peso seco/ agua destilada fue de 1:10.

Obtención de partículas: Las 20 trozas se astillaron en un astillador industrial de disco vertical y finalmente se virutearon en un equipo Pallmann PZ2 de laboratorio. El material fue clasificado por tamaño para las capas externas e internas del tablero. Las partículas fueron secadas a un 6,1\% para la capa externa y un $5,4 \%$ para la capa media.

Fabricación de Tableros. Se fabricaron tableros con cuatro densidades nominales diferentes; 550 $\mathrm{kg} / \mathrm{m}^{3} ; 600 \mathrm{~kg} / \mathrm{m}^{3} ; 650 \mathrm{~kg} / \mathrm{m}^{3}$ y $700 \mathrm{~kg} / \mathrm{m}^{3}$. Los tableros, 4 por cada densidad, tenían tres capas, dos externas de partículas finas y una media de partículas gruesas. Otras condiciones de fabricación fueron las siguientes:

Espesor: $16 \mathrm{~mm}$. 
Proporción capa externa / capa interna: 40/60

Factor de encolado capa externa: $10 \%$

Factor de encolado capa interna: 6,5\%

Catalizador: aplicado en capa interna: $1 \%$

Presión máxima: $4,5 \mathrm{~N} / \mathrm{mm}^{2}$

Temperatura de prensado: $190{ }^{\circ} \mathrm{C}$

Factor de tiempo de prensado: $15 \mathrm{~s} / \mathrm{mm}$

Propiedades. Los ensayos físicos y mecánicos, se realizaron de acuerdo con las normas y métodos que se especifican a continuación:

- Geometría y tamaño de las partículas, según Poblete (1979).

- Densidad de tablero (DIN 52361). (352 probetas)

- Tracción perpendicular (EN 319). (224 probetas)

- Flexión estática, MOR y MOE (EN 310). (128 probetas)

- Hinchamiento a 2 y $24 \mathrm{~h}$ (EN 317). (128 probetas)

- Absorción de agua a 2 y 24 h. En 128 probetas según EN 317 y con la metodología de ASTM 1037. Las mediciones de la absorción de agua se realizaron de forma paralela a las de hinchamiento.

Para lograr una correlación adecuada entre densidad del tablero y las propiedades, se determinó la densidad de cada probeta y este valor junto al de la propiedad medida. Para los análisis estadísticos la densidad se consideró la variable independiente y la propiedad física o mecánica la dependiente. Con estos datos se realizaron análisis de correlación y de varianza.

\section{RESULTADOS Y DISCUSIÓN}

\section{Caracterización de la madera y partículas utilizadas}

Densidad de la madera. El promedio de densidad básica de la madera fue de $440 \mathrm{~kg} / \mathrm{m}^{3}$, similar al que se registra en especies nativas latifoliadas que se procesan en este tipo de industrias. $\mathrm{Al}$ comparar la densidad de la periferia con la del centro del trozo, se verificó que existen diferencias, bajando la densidad hacia la médula de $470 \mathrm{~kg} / \mathrm{m}^{3}$ a $410 \mathrm{~kg} / \mathrm{m}^{3}$.

Los valores obtenidos de los 10 árboles analizados, permiten aseverar que se trata de una materia prima apta para la fabricación de tableros de partículas, ya que produciendo tableros con las densidades que actualmente se comercializan en Chile $\left(650 \mathrm{~kg} / \mathrm{m}^{3}\right)$ se lograrían razones de compresión entre 1,6 y 1,4 .

Valor de pH de las partículas. Los valores obtenidos y parámetros estadísticos básicos se presentan en la tabla 1. 
Tabla 1: Valor de $\mathrm{pH}$ de las partículas de Eucalyptus nitens

\begin{tabular}{|l|c|c|c|c|}
\hline \multirow{2}{*}{\multicolumn{1}{|c|}{ Parámetro }} & \multicolumn{2}{|c|}{ Capa externa } & \multicolumn{2}{c|}{ Capa media } \\
\cline { 2 - 5 } & $\begin{array}{c}\text { Mezcla con } \\
\text { agua destilada }\end{array}$ & $\begin{array}{c}\text { Filtrado sin } \\
\text { partículas }\end{array}$ & $\begin{array}{c}\text { Mezcla con } \\
\text { agua destilada }\end{array}$ & $\begin{array}{c}\text { Filtrado sin } \\
\text { partículas }\end{array}$ \\
\hline Promedio & 4,78 & 4,52 & 4,82 & 4,56 \\
\hline Desviación Estándar & 0,18 & 0,01 & 0,32 & 0,21 \\
\hline Mínimo & 4,61 & 4,51 & 4,36 & 4,27 \\
\hline Máximo & 4,98 & 4,54 & 5,13 & 4,83 \\
\hline
\end{tabular}

Los valores promedio de $\mathrm{pH}$ se asemejan a los de Pinus radiata, que es la madera utilizada en la fabricación de tableros de partículas en Chile (Poblete y Sanchez 1991). El pH ácido registrado permite deducir que la madera de E. nitens debería facilitar un buen fraguado de la urea formaldehido.

Geometría de las partículas. Esta caracterización está dada por el valor promedio del largo, espesor y coeficiente de esbeltez (C.E.). Los valores de estas dimensiones se presentan en la tabla 2. Los resultados demuestran que la participación en la capa media está dada en su mayoría por partículas en el rango $>4,0 \mathrm{~mm}$, mientras que para la capa externa la mayor participación se da en el rango 2,0 - 1,4. Los tamaños obtenidos son los normales para especies latifoliadas procesadas con el método aplicado (Poblete 1989). La distribución de tamaños y los valores de coeficiente de esbeltez demuestran que el proceso de corte se ha desarrollado de forma semejante a la que se da con otras especies.

Tabla 2: Geometría de las partículas de Eucalyptus nitens

\begin{tabular}{|c|c|c|c|c|c|}
\hline $\begin{array}{c}\text { Tipo de } \\
\text { partícula }\end{array}$ & $\begin{array}{c}\text { Rango de } \\
\text { tamaño } \\
(\mathbf{m m})\end{array}$ & Participación $(\boldsymbol{\%})$ & $\begin{array}{c}\text { Largo } \\
\text { promedio } \\
(\mathbf{m m})\end{array}$ & $\begin{array}{c}\text { Espesor } \\
\text { promedio } \\
(\mathbf{m m})\end{array}$ & C.E. promedio \\
\hline \multirow{5}{*}{ Capa Interna } & $>4,0$ & 53,4 & 34,4 & 0,5 & 66,1 \\
\cline { 2 - 6 } & $4,0-3,0$ & 19,9 & 22,8 & 0,5 & 45,6 \\
\cline { 2 - 6 } & $3,0-2,0$ & 15,4 & 19,7 & 0,5 & 39,5 \\
\cline { 2 - 6 } & $2,0-1,4$ & 4,4 & 19,0 & 0,5 & 42,1 \\
\cline { 2 - 6 } & $1,4-1,0$ & 3,9 & 7,2 & 0,3 & 24,1 \\
\cline { 2 - 6 } & $1,0-0,5$ & 2,5 & 4,8 & 0,2 & 22,0 \\
\cline { 2 - 6 } & Polvo & 0,6 & 0,0 & 0,0 & 1,0 \\
\cline { 2 - 6 } & Promedios & & 27,2 & 0,5 & 53,4 \\
\hline \multirow{5}{*}{ Capa Externa } & $>4,0$ & 3,1 & 27,2 & 0,5 & 36,3 \\
\cline { 2 - 6 } & $4,0-3,0$ & 15,8 & 23,8 & 0,4 & 31,7 \\
\cline { 2 - 6 } & $3,0-2,0$ & 22,7 & 18,3 & 0,4 & 32,0 \\
\cline { 2 - 6 } & $2,0-1,4$ & 25,6 & 17,3 & 0,4 & 44,6 \\
\cline { 2 - 6 } & $1,4-1,0$ & 11,6 & 11,7 & 0,3 & 29,8 \\
\cline { 2 - 6 } & $1,0-0,5$ & 13,8 & 5,2 & 0,1 & 20,6 \\
\cline { 2 - 6 } & Polvo & 7,5 & 0,0 & 0,0 & 1,0 \\
\cline { 2 - 6 } & Promedios & & 15,2 & 0,4 & 37,0 \\
\hline \multirow{5}{*}{} & & & & \\
\hline
\end{tabular}

C.E.: Coeficiente de esbeltez 


\section{Propiedades físicas de los tableros}

Las 128 probetas analizadas para las propiedades físicas registraron densidades de tablero que variaron entre 468 y $725 \mathrm{~kg} / \mathrm{m}^{3}$.

Hinchamiento. Los valores de hinchamiento y su relación con la densidad de los tableros, se presentan en la figura 1.

Es importante señalar que en los tableros de E. nitens fabricados con las condiciones ya especificadas, se encuentra una tendencia a aumentar el hinchamiento con aumentos de la densidad del tablero.

La norma alemana DIN 68761-1 fija los requisitos para tableros de partículas de uso general, e indica que el hinchamiento luego de 2 horas de inmersión no debe ser mayor a $8 \%$. De acuerdo con estas exigencias ninguna de las densidades ensayadas cumple con la norma. Debe tenerse presente que los tableros confeccionados no contienen productos hidrófobos, que mejoren esta propiedad. En otros estudios con E. nitens, que incluyen hidrófobos, se ha determinado un hinchamiento menor y que cumple con la norma (Gorrini et al. 2004).

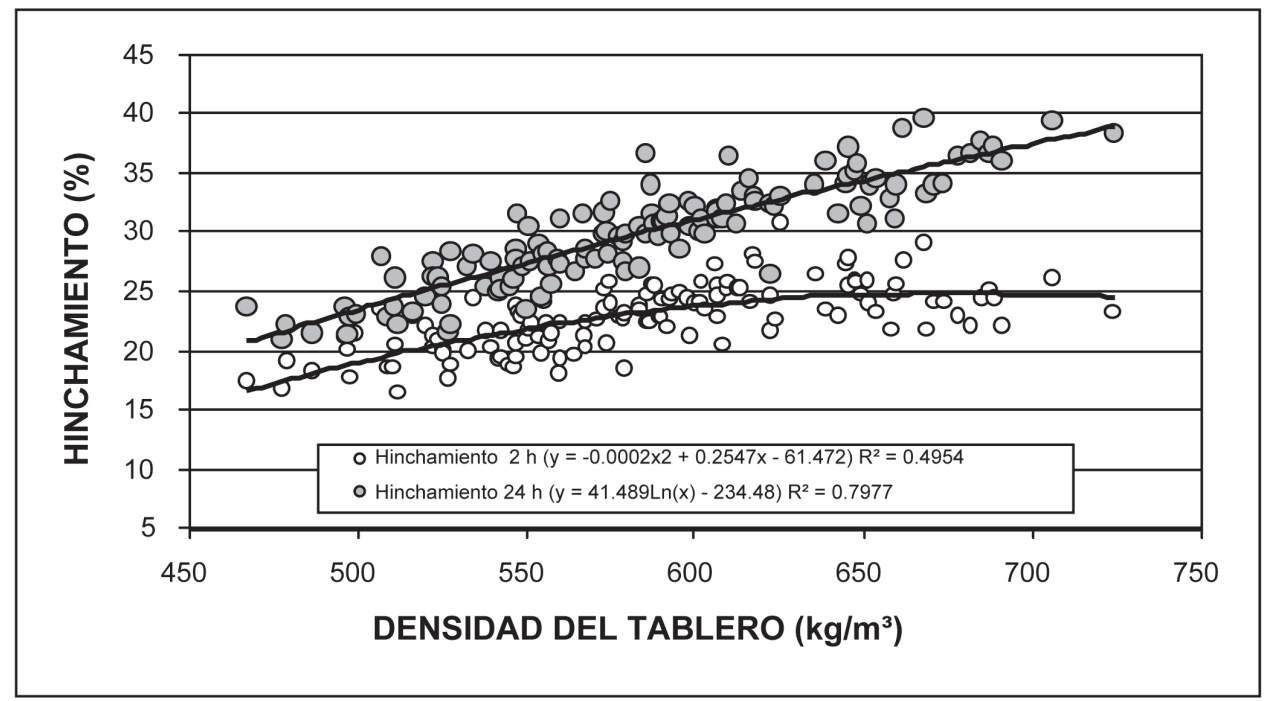

Figura 1. Efecto de la densidad de los tableros sobre el hinchamiento ( 2 y 24 horas en agua) en tableros de partículas con madera de E. nitens.

Absorción de agua. Los resultados (figura 2) muestran que el aumento de la densidad provoca una disminución en la absorción de agua, tanto para una inmersión de 2 horas como para 24 horas. Tendencias similares han sido encontradas por otros autores con otras especies (Poblete y Peredo 1989). Los tableros con una menor densidad presentan mayor espacio dentro de su estructura lo que permite captar una mayor cantidad de agua, provocando un aumento del peso.

Los coeficientes de correlación son altos $(>0,85)$ y pese a que para la absorción no existen exigencias en las normas, al comparar los valores determinados con los registrados en otras latifoliadas creciendo en Chile se comprueba que las cifras son similares (Poblete y Peredo 1989). 


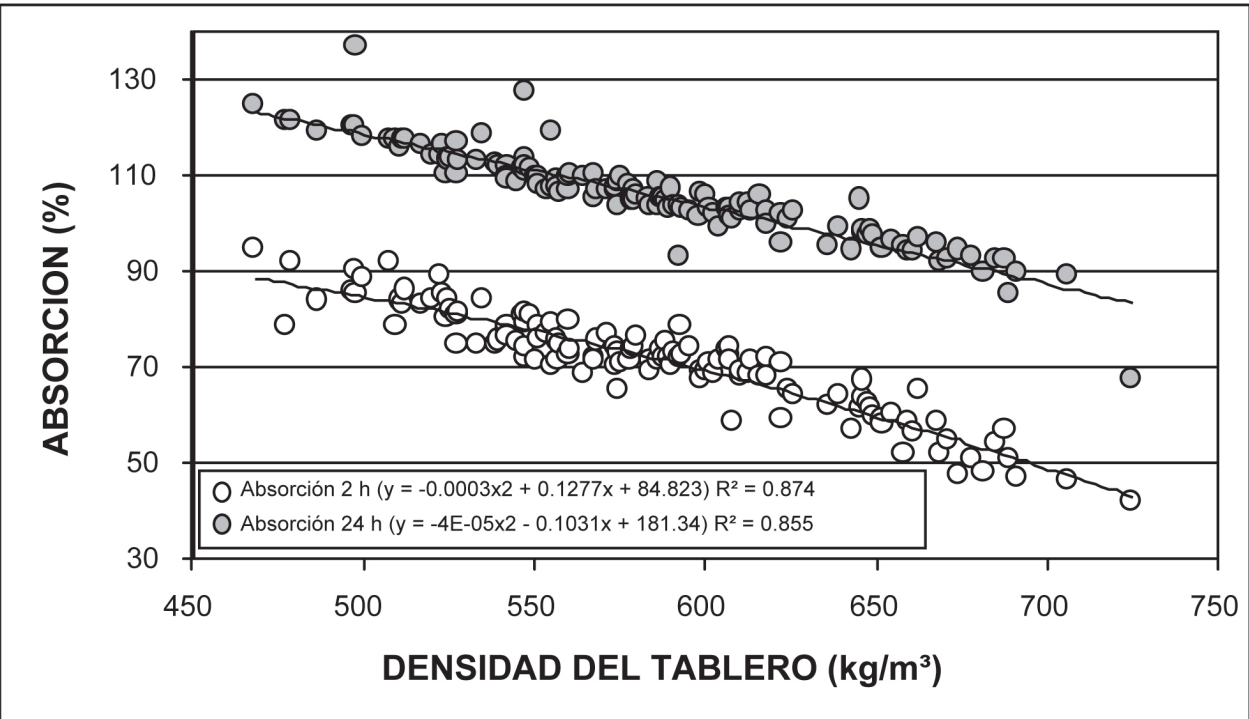

Figura 2. Efecto de la densidad del tablero en la absorción de agua (inmersión en agua por 2 y 24 horas) de tableros de partículas con madera de E. nitens.

\section{Propiedades mecánicas}

Resistencia a la flexión. En la figura 3 se presenta el desarrollo de la regresión para la relación entre densidad de tablero y módulo de rotura.

En la figura 3 es posible verificar que los valores MOR aumentan a medida que se incrementa la densidad del tablero. El valor mínimo de módulo de rotura fue de $10,7 \mathrm{~N} / \mathrm{mm}^{2}$ y alcanzó $24,7 \mathrm{~N} / \mathrm{mm}^{2}$ con una densidad de $725 \mathrm{~kg} / \mathrm{m}^{3}$.

La norma DIN 68.761-1 exige en MOR un valor mínimo de $16 \mathrm{~N} / \mathrm{mm}^{2}$. En el caso de fabricar tableros de $E$. nitens con las condiciones de este estudio, la exigencia de la norma obliga a fijar una densidad de tablero mínima de aproximadamente $580 \mathrm{~kg} / \mathrm{m}^{3}$. La norma EN 312-3 exige sólo $13 \mathrm{~N} / \mathrm{mm}^{2}$, con lo que la densidad se podría disminuir.

La tendencia encontrada para el MOE es similar, a medida que aumenta la densidad del tablero aumenta el módulo de elasticidad. Este resultado se presenta en la figura 4. 


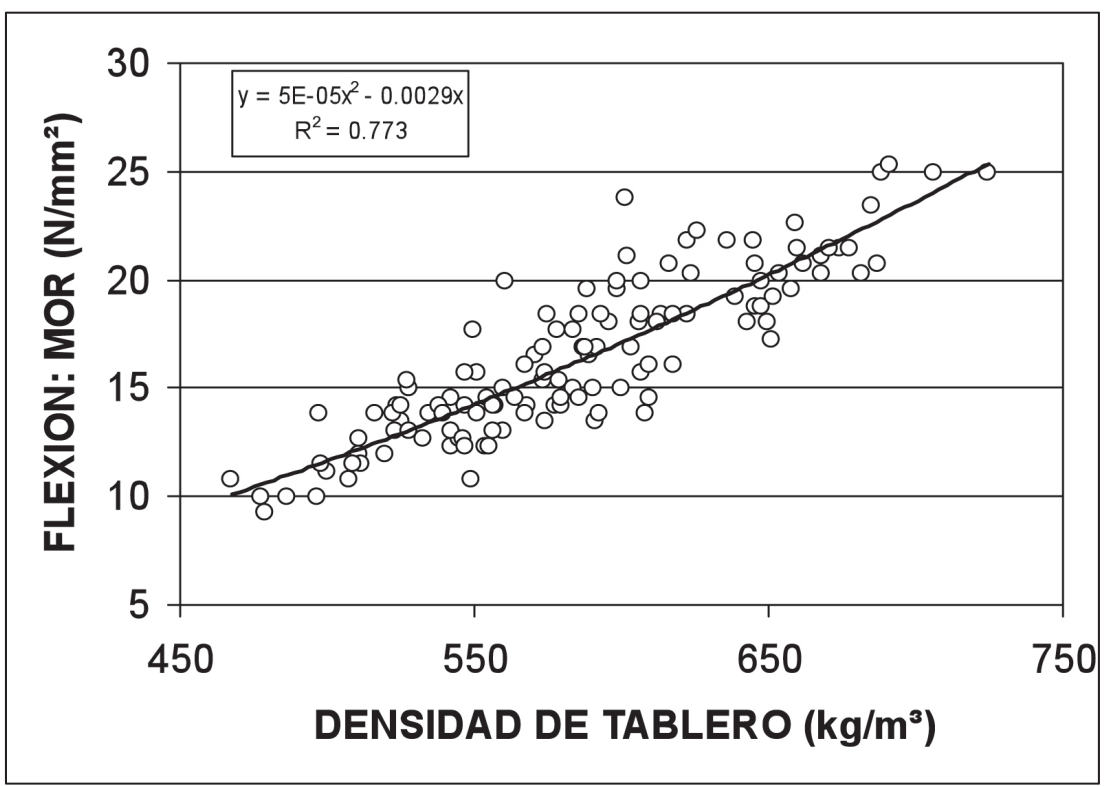

Figura 3. Efecto de la densidad del tablero en la resistencia a la flexión, MOR, en tableros de partículas con madera de E. nitens.

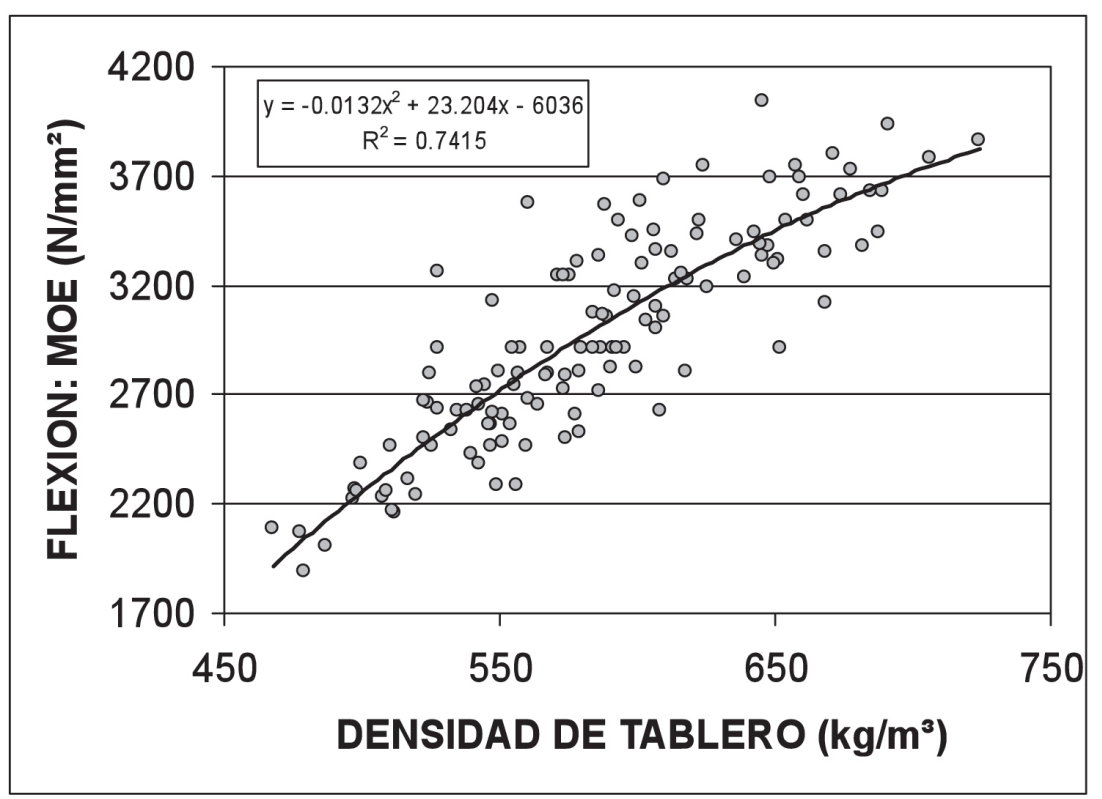

Figura 4. Efecto de la densidad del tablero en la resistencia a la flexión, MOE, en tableros de partículas con madera de E. nitens.

La norma EN 312-3 establece los requisitos para tableros a emplear en aplicaciones de interior en ambiente seco. En esta norma se especifica que el MOE de los tableros con espesores de 13 a $20 \mathrm{~mm}$ debe ser igual o superior a $1600 \mathrm{~N} / \mathrm{mm}^{2}$. Se comprueba que, con esta exigencia, todos los tableros 
cumplen con la norma.

Tracción perpendicular al plano del tablero. Esta propiedad está directamente relacionada con la calidad de la unión de las partículas en la capa central del tablero. Los resultados del estudio demuestran que la tracción aumenta a medida que aumenta la densidad del tablero (figura 5)

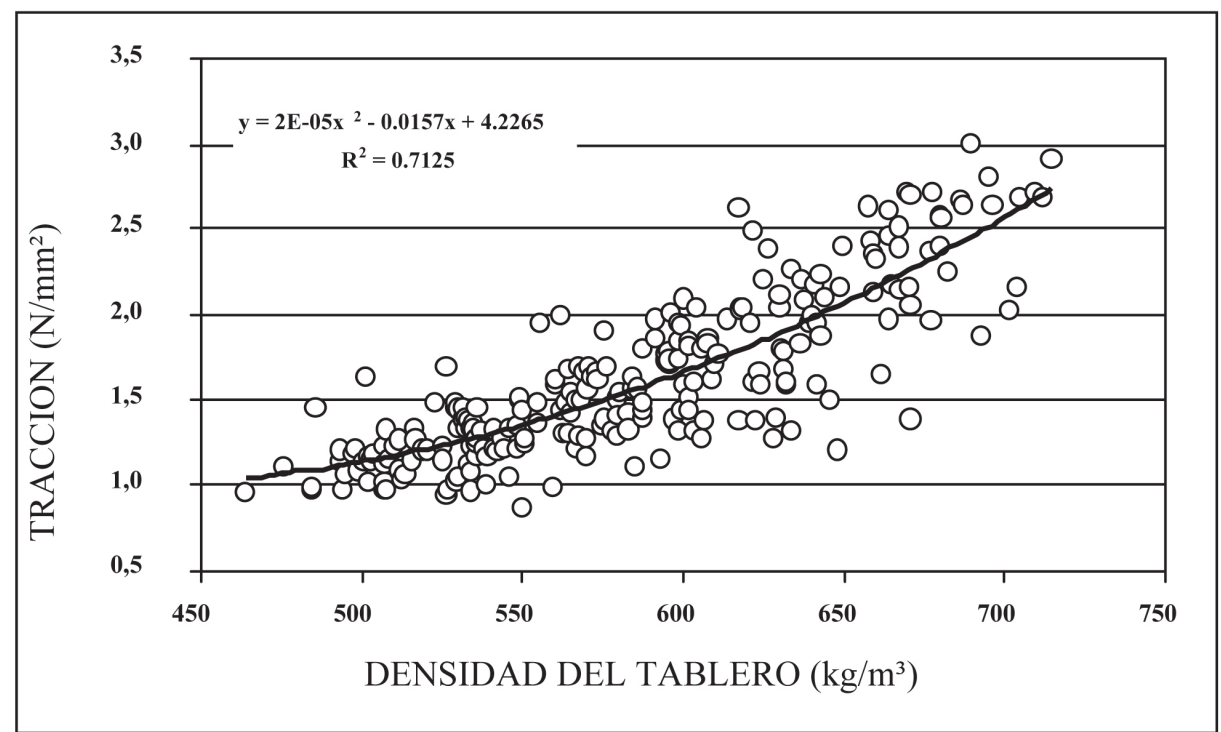

Figura 5. Resistencia a la tracción perpendicular al plano según la densidad del tablero

Los tableros fabricados con E. nitens cumplen tanto con la norma DIN 68761-1 como con la norma EN 312-3, las que exigen un mínimo de $0,35 \mathrm{~N} / \mathrm{mm}^{2}$. El valor más bajo de tracción medido en las probetas de $E$. nitens es de $0,86 \mathrm{~N} / \mathrm{mm}^{2}$.

\section{CONCLUSIONES}

Las características de las partículas utilizadas, densidad, $\mathrm{pH}$, dimensiones y geometría, confirman la aptitud de Eucalyptus nitens como materia prima para la fabricación de tableros de partículas.

El análisis de correlación entre las propiedades dependientes (hinchamiento, absorción, flexión y tracción) y la densidad del tablero dio coeficientes de correlación mayores a 0,7 , lo que permite conocer con certeza el comportamiento de esta madera en la fabricación de tableros de partículas para interiores secos.

La densidad es una variable importante de decisión al momento de proyectar las propiedades físico y mecánicas en la fabricación de los tableros de partículas de Eucalyptus nitens.

Los resultados obtenidos indican que la propiedad crítica es el hinchamiento, por lo que resulta indispensable agregar un hidrófobo y un catalizador en el adhesivo de la capa central. 
Si no se considera el hinchamiento, la flexión en su módulo de rotura (MOR) es el parámetro limitante. Los resultados indican que no se puede cumplir con la norma si se fabrican tableros con densidades inferiores a $580 \mathrm{~kg} / \mathrm{m}^{3}$.

En general, los resultados demuestran que la madera de Eucalyptus nitens es apta para ser utilizada en la fabricación de tableros de partículas.

\section{BIBLIOGRAFÍA}

ASTM 1037-99. 1999. Standard Test Methods for Evaluating Properties of Wood Fiber and Particle Panel Materials.

DIN 52182. 1994. Prüfung von Holz; Bestimmung der Rohdichte. Taschenbuch 31. Normen über Holz. Beuth-Verlag. 2 p.

DIN 52361. 1994. Bestimmung der Abmessungen, der Rohdichte und des Feuchtigkeitsgehaltes. Taschenbuch 60. Holzfasserplatten, Spanplatten, Sperrholz. Beuth-Verlag. pp. 23-25.

DIN 68761-1. 1994. Flachpressplatten für allgemeine Zwecke. FPY-Platte. Taschenbuch 60. Holzfasserplatten, Spanplatten, Sperrholz. Beuth-Verlag. pp 154-155.

EN 310. 1994. Tableros derivados de madera. Determinación del módulo de elasticidad en flexión y de la resistencia a la flexión. AENOR 1994. 11 p.

EN 312-3. 1994. Tableros de partículas. Especificaciones. Parte 3: Especificaciones de los tableros para aplicaciones de interior (incluyendo mobiliario) en ambiente seco. AENOR 1994. 10 p.

EN 317. 1994. Tableros de partículas y tableros de fibras. Determinación de la hinchazón en espesor después de inmersión en agua. AENOR 1994. 9 p.

EN 319. 1994. Tableros de partículas y tableros de fibras. Determinación de la resistencia a la tracción perpendicular a las caras del tablero. AENOR 1994. $10 \mathrm{p}$.

Gorrini, B.; Poblete H.; Hernández, G.; Dunn, F. 2004. Tableros de partículas y MDF de Eucalyptus nitens: Ensayos a escala industrial. Bosque 25(3): 89-97.

Kelly, M. 1977. Critical literature review of relationships between processing parameters and physical properties of particleboard. U.S. Gov. Printing Office. General Technical Report FPL-10. 64 p

Kollmann, F.; Kuenzi, E.; Stamm, A. 1975. Principles of wood science and technology. II. Wood Based Materials. Berlin: Ed. Springer Verlag. 672 p.

Moslemi, A. 1974. Particleboard. Vol.2: Materials. Southern Illinois University Press. 245p

Poblete, H. 1979. Inclusión de aserrín en tableros de partículas. Bosque 3(1): 38-46.

Poblete H. 1989. Tableros de partículas con renovales de roble (Nothofagus obliqua), raulí (N. alpina) y un híbrido de ambos. Bosque 10(1): 9-17.

Poblete, H.; Sanchez, J. 1991. Tableros con corteza de Pinus radiata. Bosque 12(1): 17-26.

Poblete, H.; Loncomilla, E.; Inzunza, L. 2000. Densidad del tablero de partículas y estabilidad 
dimensional. Bosque 21(2): 69-83.

Poblete, H.; Peredo, M. 1989. Tableros de desechos del debobinado de especies chilenas. Bosque 11(2): 45-58.

Poblete, H. 2001. Tableros de Partículas. Chile: Ed. El Kultrún. 177 p.

Poblete, H. 2002. Estudio técnico de la utilización de Eucalyptus nitens en la fabricación de contrachapados: ensayo con trozas basales y segundas. Informe de Convenio. UACh - Forestal Mininco S.A Valdivia, Chile. Facultad de Ciencias Forestales. Universidad Austral de Chile. 42 p.

Poblete, H. 2004. El nitens puede ser la especie latifoliada industrial del futuro. Lignum 14(73): 3032.

Roffael, E.; Poblete, H.; Torres, M. 2000. Über die acidität von Kern- und Splintholz der Kastanie (Castanea sativa) aus Chile. Holz als Roh- und Werkstoff 58: 120-122.

Stegmann, G.; Durst, J. 1964. Spanplatten aus Buchenholz. Holz-Zentralblatt 90(153): 19.

Stewart, H.A.; Lehmann, W.F. 1973. High-quality particleboards from cross grain, knife planed hardwood flakes. Forest Products Journal 23(8): 52-60.

Vásquez, C. 2005. Eucalyptus nitens: Mucho más que sólo una opción pulpable. Lignum 15(80): $52-55$.

Vital, B.; Lehmann, W.; Boone, R. 1974. How species and board densities affect properties of exotic hardwood particleboards. Forest Product Journal 24(12): 37- 45. 